\title{
Homogeneity in donkey sentences *
}

\author{
Lucas Champollion \\ New York University
}

\begin{abstract}
Donkey sentences have existential and universal readings, but they are not often perceived as ambiguous. I extend the pragmatic theory of homogeneity in plural definites by Križ (2016) to explain how context disambiguates donkey sentences. I propose a semantic theory that produces truth value gaps in certain scenarios, and a pragmatic theory that fills these gaps in context-dependent ways. By locating the parallel between donkey pronouns and definite plurals in the pragmatics rather than in the semantics, I avoid problems known to arise for some previous accounts according to which donkey pronouns and definite plurals both have plural referents (Krifka 1996; Yoon 1996). I sketch an extension of plural compositional DRT (Brasoveanu 2008) that delivers the required truth value gaps by building on concepts from error-state semantics and supervaluation quantifiers.
\end{abstract}

Keywords: donkey sentences, trivalence, weak/strong (existential/universal) ambiguity, extension gaps, pragmatics

\section{Introduction}

It is an old observation that some donkey pronouns seem to be understood as having existential force and others as having universal force. The following pair is adapted from Yoon 1996:

(1) Usually, if a man has a garage with a window, ...

a. he keeps it open while he is away.

b. he keeps it closed while he is away.

On the most plausible reading of (1a), the donkey pronoun it could be paraphrased as one of the windows in his garage (except that there is no implication that the

* I am indebted to Robert Henderson for extensive discussions of the contents of this paper and for specific and very helpful suggestions regarding the implementation of error state semantics and supervaluation quantifiers in PCDRT. I am grateful to Chris Barker, Justin Bledin, Adrian Brasoveanu, Dylan Bumford, Jan van Eijck, Makoto Kanazawa, Manuel Križ, Sophia Malamud, Philippe Schlenker, Anna Szabolcsi, the NYU semantics group, and the audience at SALT for helpful feedback. All errors are mine. 
garage actually has more than one window). This is sometimes referred to as a weak or existential interpretation; following Chierchia (1992, 1995), I will call it the $\exists$-reading. As for (1b), on its most plausible reading, the meaning of the pronoun is paraphrasable as all of the windows in his garage. This is the strong or universal interpretation, and I will refer to it as the $\forall$-reading. ${ }^{1}$

Yoon $(1994,1996)$ and Krifka (1996) link this behavior of donkey pronouns to maximal and nonmaximal interpretations of plural definites. Imagine the following sentences, adapted from Krifka 1996, uttered in a situation where the local bank has a safe that is accessible through any one of three doors.

(2) (I could not reach the safe because ...)

a. The doors are closed.

b. The doors are open.

As Krifka observes, in the situation just described, sentence (2a) expresses the fact that all of the doors are closed (a maximal interpretation), while sentence (2b) expresses the fact that at least some of the doors are open (a nonmaximal interpretation). These two readings naturally correspond to the $\forall$-reading and to the $\exists$-reading of donkey pronouns. On the basis of this kind of similarity, Yoon and Krifka develop a sum-based analysis of donkey sentences, in which the pronoun it in (1) is analyzed as referring to the mereological sum of all the windows in the garage in question. It is interpreted as number-neutral, that is, it does not presuppose that there is more than one window or door. Apart from this, it is essentially synonymous with the definite plural the doors in (2).

However, Kanazawa (2001) convincingly shows that singular donkey pronouns, unlike definite plurals, cannot refer to sums. For example, singular donkey pronouns are incompatible with collective predication, while definite plurals are compatible:

a. Every donkey-owner gathers his donkeys at night.

b. *Every farmer who owns a donkey gathers it at night.

This poses a challenge for analyses of the $\exists / \forall$ ambiguity that try to reduce the behavior of donkey pronouns to that of plural definite descriptions. The goal of this paper is to develop a theory that meets this challenge but succeeds at predicting how context disambiguates donkey sentences. To do so, I build on a pragmatic account of how context disambiguates plural definites. I adopt the specific implementation in Križ 2016; for a similar and more elaborate account, see Malamud 2012. To avoid the problems that arise from interpreting pronouns as referring to sums, I locate the

1 As Kanazawa (1994) notes, the weak/strong terminology is misleading, because when the embedding quantifier is downward entailing in its nuclear scope, as in the case of no, the weak reading is the logically stronger of the two. 
parallel between donkey pronouns and definite plurals in the pragmatics rather than in the semantics. My core strategy, following a suggestion by Kanazawa (1994), is to combine a trivalent semantics that produces truth-value gaps in certain cases with a pragmatics that fills these gaps with truth or falsity. This corresponds to what Malamud (2012), Križ (2016), and others assume for plural definites. I assume that these truth-value gaps are filled at the sentence level, not at the level of plural definites or donkey pronouns. Donkey pronouns are not similar to plural definites; it is donkey sentences as a whole that are similar to sentences with plural definites.

The paper is structured as follows. Section 2 highlights the pragmatic nature of the $\exists / \forall$ ambiguity by focusing on the role of context in disambiguating it. Section 3 is a brief summary of the theory developed by Križ (2016) for plural definites. Section 4 applies this theory to donkey sentences. Section 5 compares the present account with previous work. Section 6 sketches a compositional semantics that delivers truth-value gaps as needed by building on plural compositional discourse representation theory (Brasoveanu 2008, 2010). Section 7 concludes.

\section{The $\exists / \forall$ ambiguity and the role of context}

It is easy to judge the donkey sentence (4) if no man treats any two donkeys differently: In such scenarios, if every man beats every donkey he owns, it is clearly true, and if some donkey-owner beats no donkey he owns, it is clearly false.

(4) Every man who owns a donkey beats it.

Truth conditions become more difficult to ascertain in scenarios I will call mixed, namely those where every man owns and beats one donkey, and at least some men own additional donkeys that they do not beat (e.g. Heim 1982; Rooth 1987).

I will say that a donkey sentence has a heterogeneous interpretation if it is readily judged true in relevant mixed scenarios; otherwise, I will speak of homogeneous interpretations. An example whose most salient interpretation is homogeneous is (5a), adapted from Rooth 1987. It is homogeneous because it is judged false as soon as someone lets his 10-year-old son drive the car, even if he has another 10-year-old son that he forbids from driving it. Two heterogeneous examples are (5b), adapted from Schubert \& Pelletier 1989, and (5c), from Chierchia 1995.

a. No man who has a 10-year-old son lets him drive the car.

b. Usually, if a man has a quarter in his pocket, he will put it in the meter.

c. No man who has an umbrella leaves it home on a day like this.

As for (4) itself, Chierchia (1995) reports that although it prima facie seems to be most naturally analyzable in terms of a (homogeneous) $\forall$-reading, it turns out to 
allow quite clearly for (heterogeneous) $\exists$-readings in suitable contexts. Chierchia gives this context as a tongue-in-cheek example, attributed to Paolo Casalegno:

(6) The farmers of Ithaca, N.Y., are stressed out. They fight constantly with each other. Eventually, they decide to go to the local psychotherapist. Her recommendation is that every farmer who has a donkey should beat it, and channel his/her aggressiveness in a way which, while still morally questionable, is arguably less dangerous from a social point of view. The farmers of Ithaca follow this recommendation and things indeed improve.

The distinction between homogeneous and heterogeneous readings cuts across the one between $\exists$-readings, such as (5a) and (5b), and $\forall$-readings, such as (5c). It also cuts across the distinction between determiner-based donkey sentences, such as (5a) and (5c), and adverbial ones, such as (5b), and across the one between downward-entailing embedding quantifiers, as in (5a) and (5c), and upward-entailing ones, as in (5b). Hence it is not possible to reduce one of these distinctions to another.

The influence of context on donkey sentences has been noticed before:

Anyone who catches a Medfly should bring it to me.

Gawron, Nerbonne \& Peters (1991) observe that the interpretation of (7) is different depending on whether the speaker is a biologist looking for samples on a field trip, in which case the $\exists$-reading emerges, or a health department official engaged in eradicating the Medfly, in which case the $\forall$-reading surfaces.

\section{Križ (2016) on plural definites}

Sentences with definite plurals exhibit a related phenomenon also called homogeneity, whose presence likewise depends on the context (e.g. Löbner 2000; Križ 2016). As mentioned before, if I can reach a safe by going through any one of three doors arranged in parallel, and if two of these doors are open, the sentence The doors are open is readily judged true. Hovewer, if the doors are arranged in a sequence and one needs to pass through all of them, the sentence is only judged true if all the doors are open. If some but not all of them are open, it is judged false or neither true nor false.

To explain how these different interpretations arise, Križ (2016) assumes a salient current issue $I$, a partition of the set of worlds which gives rise to an equivalence relation $\approx_{I}$. Intuitively, $w \approx_{I} w^{\prime}$ means that the current issue is resolved in the same way in $w$ and $w^{\prime}$, and any differences between these two worlds are irrelevant for current purposes. A sentence $S$ is judged true just in case it is true enough at $w$ with respect to $I$, where being "true enough" means being true either at $w$ itself or at some 
$w^{\prime} \approx_{I} w$ (see Lewis 1979; Lasersohn 1999; Malamud 2012). ${ }^{2}$

Križ assumes that sentences can have extension gaps (van Fraassen 1969; Schwarzschild 1993). In a scenario when some but not all doors are open, The doors are open is literally (at the semantic level) neither true nor false. These literal truth values are not intended to directly reflect native speakers' intuitions. They are merely an intermediate step on the way towards computing pragmatic truth values.

Križ proposes to relax the Gricean Maxim of Quality in the following way. A sentence $S$ may be used at $w$ to address an issue $I$ even if it lacks a truth value at $w$, as long as it is true enough at $w$ and not false at any $w^{\prime} \approx_{I} w$. That is, speakers may utter a sentence even if they do not believe it to be true, as long as they do not believe it to be false at any world that is equivalent to the actual world. ${ }^{3}$ Sentences that are true enough are judged true. In the absence of a current issue, sentences that are neither true nor false at the semantic level cannot be assigned a pragmatic truth value. Speakers who are presented with such sentences and scenarios can try to guess what the current issue might; when no issue can be easily guessed, speakers may become confused and give guarded judgments.

Suppose that the current issue is whether there is a way to the safe. That is, suppose that $w^{\prime} \approx_{I} w$ just in case the safe is reachable either in both $w$ and $w^{\prime}$ or in neither $w$ nor $w^{\prime}$. Say the doors are arranged in parallel. Consider two worlds $w_{\text {all }}$, where all the doors are open, and $w_{\text {some }}$, where two of three doors are open (a mixed scenario). These worlds are equivalent for current purposes, and The doors are open counts as true enough at both of them. Accordingly, it will be interpreted non-maximally (and hence, heterogeneously) as the proposition $\left\{w_{\text {all }}, w_{\text {some }}\right\}$. Now consider a context where the doors are arranged in sequence: $w_{\text {all }}$ and $w_{\text {some }}$ are no longer equivalent. Instead, $w_{\text {some }}$ is equivalent to a world $w_{\text {none }}$ where no door is open. Since $w_{\text {all }}$ is the only world at which The doors are open is true enough, it is interpreted maximally (and hence homogeneously) as $\left\{w_{\text {all }}\right\}$.

\section{Applying Križ 2016 to donkey sentences}

Let us assume that donkey sentences have extension gaps at worlds that correspond to mixed scenarios. Then we can apply this theory straightforwardly. Suppose the semantics assigns sentence (4), repeated here, the literal truth conditions below:

(8) Every man who owns a donkey beats it.

2 Current issues are similar to questions under discussion (Roberts 2012) and to the way questions are modeled in Groenendijk \& Stokhof 1984. Nevertheless, Križ (2016: Section 4.5) resists identifying current issues with questions under discussion because it is not possible to directly manipulate the current issue by asking it as a question. I will remain neutral on how these two concepts are related.

3 More generally, Križ stipulates that $S$ may not be used to address an issue $I$ if there are $w_{1}$ and $w_{2}$ such that $w_{1} \approx_{I} w_{2}$ and $S$ is true at $w_{1}$ but false at $w_{2}$, whether the actual world is among them or not. 
Homogeneity in donkey sentences

a. true iff every donkey-owner beats every donkey he owns;

b. false iff at least one donkey-owner does not beat any donkey he owns;

c. neither in all other cases, in particular, if every donkey-owner beats exactly one donkey and one of them owns a donkey he does not beat.

For the purpose of exposition, pretend that there are only three possible worlds. Let $w_{\text {true }}$ be a world where (8a) holds, $w_{\text {false }}$ one where ( $8 \mathrm{~b}$ ) holds, and $w_{\text {mixed }}$ one where (8c) holds. Assume that the current issue in scenario (6) is whether every farmer follows the recommendation to beat at least one donkey. Then $w_{\text {true }} \approx_{I} w_{\text {mixed }}$. Hence (4) is interpreted as $\left\{w_{\text {true }}, w_{\text {mixed }}\right\}$; this is a heterogeneous $\exists$-reading. If we change the scenario so that the recommentation is to beat all one's donkeys, $w_{\text {mixed }}$ and $w_{\text {false }}$ are now equivalent to each other, but not to $w_{\text {true }}$. This time, (4) is not true enough at $w_{\text {mixed }}$. It is pragmatically interpreted as $\left\{w_{\text {true }}\right\}$. Since this proposition does not contain $w_{\text {mixed }}$, sentence (4) receives a homogeneous reading; and since at $w_{\text {true }}$, every donkey-owner beats all of his donkeys, this is a $\forall$-reading.

Turning to sentences headed by no, we have seen that sentence $(5 \mathrm{c})$ has an $\forall$-reading. Assume that it has the following literal truth conditions:

(9) No man who has an umbrella leaves it home on a day like this. $=(5 \mathrm{c})$

a. true iff no umbrella-owner leaves any of his umbrellas home;

b. false iff at least one umbrella-owner leaves all his umbrellas home;

c. neither in all other cases, in particular, if every umbrella-owner takes exactly one umbrella along, and someone also leaves one home.

As before, let $w_{\text {true }}, w_{\text {false }}$ and $w_{\text {mixed }}$ be worlds in which (9a), (9b), and (9c) are the case respectively. Suppose that the current issue is whether any man with an umbrella is getting wet. A man gets wet if he fails to take any umbrella along. This is the case at $w_{\text {false }}$. It is neither the case at $w_{\text {true }}$ nor at $w_{\text {mixed }}$, so these two worlds are equivalent. Given this issue, $(5 \mathrm{c})$ is therefore true enough at both $w_{\text {true }}$ and $w_{\text {mixed }}$. Since $w_{\text {true }}$ is not equivalent to $w_{\text {false }},(5 \mathrm{c})$ can be used to address the current issue at both $w_{\text {true }}$ and $w_{\text {mixed }}$. This means $(5 \mathrm{c})$ will be pragmatically interpreted as $\left\{w_{\text {true }}, w_{\text {mixed }}\right\}$. Since this proposition contains $w_{\text {mixed }}$, this is a heterogeneous reading. Since the strongest thing we can say about both $w_{\text {true }}$ and $w_{\text {mixed }}$ is that no umbrella-owner lets all of his umbrellas home, this is a $\forall$-reading.

Now let us consider a donkey sentence headed by no that has a homogeneous reading. Assume that sentence (5a) has the following truth conditions:

(10) No man who has a 10-year-old son lets him drive the car. = (5a)

a. true iff no father lets any of his sons drive his car;

b. false iff at least one father lets all his sons drive his car;

c. neither in all other cases, for example, if every father allows one son 
to drive the car, and some of them have additional sons that they don't.

Let $w_{\text {true }}, w_{\text {false }}$ and $w_{\text {mixed }}$ match these propositions as before. Suppose that the current issue is whether there are reckless fathers. A father who allows just one of his sons to drive the car is just as reckless as one who gives permission to all of his sons. Reckless fathers are absent from $w_{\text {true }}$ but present at both $w_{\text {false }}$ and $w_{\text {mixed }}$, so $w_{\text {false }} \approx_{I} w_{\text {mixed }}$. Hence (5a) is true enough only at $w_{\text {true }}$. Since $w_{\text {true }} \not_{I} w_{\text {false }}$, (5a) can be used to address the current issue. This means (5a) will be pragmatically interpreted as $\left\{w_{\text {true }}\right\}$. Therefore, (5a) receives a homogeneous reading. Since at $w_{\text {true }}$, no father lets any $(\exists)$ of his sons drive the car, this is an $\exists$-reading. ${ }^{4}$

An interesting prediction arises from the idea that some contexts might provide fine-grained current issues in which every world represents an equivalence class of its own (up to some remote distinctions that are not at stake). We might paraphrase this as "What is the actual world like?" (see van Rooij 2003; Malamud 2012). Given such a context, our theory will map to false every world for which the semantics returns either false or neither. Given a trivalent proposition $p$, let a fact-finding context be any issue $I$ such that for all $w$ and $w^{\prime}$, if $p(w)=$ true and $p\left(w^{\prime}\right)=$ neither then $w \not_{I} w^{\prime}$. A donkey sentence that is interpreted in a fact-finding context will always be interpreted as having a homogeneous reading. For donkey sentences headed by every, this is the $\forall$-reading; for sentences headed by no, this is the $\exists$-reading. Here is a sentence that evokes a fact-finding context, from Kanazawa 1994:

(11) Every girl in this neighborhood who has a younger brother is taller than him.

As Kanazawa notes, this sentence is judged false when one girl in the neighborhood has a younger brother taller than her, even if she is taller than her other younger brothers. This is the $\forall$-reading. If we replace every in (11) by no and interpret the sentence again in a fact-finding context, the $\exists$-reading emerges, as expected.

To sum up, we have a simple pragmatic theory that expects the semantic component to pass it a trivalent proposition and a current issue (an equivalence relation over possible worlds). The theory maps the trivalent proposition to an ordinary bivalent proposition that is true in mixed scenarios whenever the current issue lumps those scenarios together with worlds at which the proposition is true.

4 Some donkey sentences are formulated in such a way as to make mixed scenarios logically or practically impossible, such as Most farmers who own exactly one donkey beat it or Most men who have a Social Security number know it by heart (see Kanazawa 1994: p. 113). For the latter sentence, the "mixed" scenarios would involve people who have more than one Social Security number (something impossible in the US American context). 
Homogeneity in donkey sentences

\section{Comparison with previous work}

The question of which factors affect the $\exists / \forall$ ambiguity has been taken on by many authors (Heim 1990; Gawron et al. 1991; Chierchia 1992, 1995; Dekker 1993; Geurts 2002). Here I focus on two proposals that are similar in spirit to mine in that they do not postulate a lexical ambiguity: Kanazawa 1994 and Barker 1996.

\subsection{Kanazawa 1994}

Kanazawa (1994) proposes a principled explanation why the $\exists$-reading and $\forall$-reading are natural interpretations of donkey sentences and what makes one or the other surface. He claims that all other things being equal, the availability of $\exists$-readings and $\forall$-readings of donkey sentences headed by a determiner is systematically related to the monotonicity properties of that determiner.

Kanazawa notes that the effect of the determiner every, at least relative to other determiners like most, no, and at least two, is to make the $\forall$-reading more readily available; in fact, his sense is that sentences with every have a default preference for the $\forall$-reading. At the same time, he acknowledges that there are clear examples of the $\exists$-reading with every as well.

We can account for Kanazawa's observation that every triggers $\forall$-readings by default by appealing to the notion of fact-finding context described in Section 4. It is natural to assume that sentences presented in absence of any clues as to what the current issue might be are typically interpreted as if they had been uttered in a fact-finding context (for discussion see van Rooij 2003; Malamud 2012).

Kanazawa discusses a generalization to the effect that determiners that are downward-entailing on their nuclear scope (such as no, few, and at most $N$ ) only have the $\exists$-reading. ${ }^{5}$ We have seen that $(5 \mathrm{c})$ is a counterexample to that generalization; Kanazawa mentions other counterexamples and notes that to the extent that intuitions are clear about them, they tend to favor the $\forall$-reading:

a. No man who had a credit card failed to use it.

b. Not all students who borrowed a book from Peter returned it.

Again, we can make sense of the tendency behind this generalization by assuming that the default context is fact-finding. The sentences in (12) are exceptions because they evoke current issues that are not fact-finding, such as: Did every card-owner pay by card? and Did Peter get all of his books back?

Kanazawa also discusses the role of context in selecting an interpretation of a given donkey sentence. He attributes this example to David Beaver (p.c.):

5 Kanazawa attributes this generalization to Rooth 1987 but notes that Rooth does not explicitly endorse it; nor does Kanazawa himself. 
(13) A: John has a silver dollar. He didn't put it in the charity box.

B: No, everybody who had a coin put it in the box.

As he notes, the context created by A's utterance makes the $\forall$-reading of B's response the only sensible interpretation. This makes sense on the present account if we assume that A's utterance gives rise to the current issue Did anybody keep any of their coins? More generally, we can recast questions about the availability of various readings as questions about the availability of various current issues.

One benefit of the theory developed here is that it accounts for the observation that "people have firm intuitions about situations where farmers are consistent about their donkey-beating" while they give "varied and guarded judgments" in mixed scenarios (Rooth 1987). In consistent situations, the semantics delivers a classical truth value, so there is no need to consider what the current issue might be. This is in line with a speculation by Kanazawa (1994):

$[\mathrm{P}]$ eople are capable of assessing the truth value of a donkey sentence without resolving the 'vagueness' of the meaning given by the grammar when there is no need to do so. For our purposes, it is enough to assume that underspecification causes no problem for people in assigning a truth value to a donkey sentence in situations where the uniqueness condition for the donkey pronoun is met. These are a special class of consistent donkey-beating situations, and the uniqueness condition can be checked just by looking at the extensions of the predicates in the N' of the sentence. (Kanazawa 1994: p. 152)

The present account extends this perspective to all consistent donkey-beating situations. Consider for example a situation where every man owns two donkeys and beats both of them. Even though the uniqueness condition for the donkey pronoun is not met, the present account still predicts that the donkey sentence (4) is true no matter what the current issue is. ${ }^{6}$

Finally, one difference between Kanazawa 1994 and the present theory concerns determiners such as several or at least $n$ that are upward-entailing on both arguments, such as Several men who had an umbrella left it at home today. Donkey sentences with such determiners are generally claimed to only have the $\exists$-reading. On the present account, the current issue determines their reading; for example, for the

6 On the present account, even some non-consistent situations are assigned a classical truth value by the semantics and are therefore not be dependent on the current issue for their interpretation. Thus if every man owns two donkeys, John beats neither of his donkeys, and everyone else beats only one of his donkeys, the semantics predicts sentence (4) to be false no matter what the current issue is. This leads us to expect that speakers should not hesitate to judge such a sentence false. I believe that this is on the whole correct; but see Kanazawa 2001: Section 6.2 for a different perspective. 
sentence just mentioned, the issue Did anyone get wet? predicts an $\forall$-reading. The monotonicity principle in Kanazawa 1994 predicts that upward-entailing determiners prefer the $\exists$-reading; an additional principle ensures that the $\forall$-reading is ruled out and not merely dispreferred. This principle is limited to intersective determiners and enforces that they have only the $\exists$-reading. As Yoon (1996) notes, this principle is problematic in the case of $n o$, which has the $\forall$-reading in sentence $(5 \mathrm{c})$.

\subsection{Barker 1996}

Barker 1996 shares many aspects and predictions of the present theory and has in part inspired it. However, it only briefly touches on donkey sentences headed by determiners. The main focus is on adverbial donkey sentences, such as these:

a. Usually, if a woman owns a dog, she is happy.

b. Usually, if an artist lives in a town, it is pretty.

c. Usually, if a linguist hears of a good job, she applies for it.

Following earlier work, Barker distinguishes between symmetric and asymmetric interpretations of donkey sentences. Sentence (14a) is naturally understood as making a claim about how many dog-owning women are happy. If a woman owns more than one dog, she is counted only once. Barker refers to this as a subject-asymmetric reading. Sentence (14b) is about the number of towns that have artists living in them (an object-asymmetric reading), and sentence (14c) is about linguist-job pairs (a symmetric reading). Barker's main claim is that asymmetrically interpreted adverbial donkey sentences come with a homogeneity presupposition:

The homogeneity hypothesis (HH, Barker 1996):

The use of a proportional adverbial quantifier when construed under a particular proportional reading presupposes that members of the same quantificational case all agree on whether they satisfy the nuclear scope.

Barker defines quantificational cases as equivalence classes of variable assignments that agree on what they assign to those variables that are bound by the adverbial quantifier. In (14a), each woman corresponds to a quantificational case. According to $\mathrm{HH}$, (14a) presupposes that any woman is happy either about all of her dogs, or about none of them. Likewise, (14b) presupposes that any town is pretty or not no matter which artists live in it. No asymmetric readings are available for (14c), because the homogeneity presuppositions of these readings fail. In effect, homogeneity presuppositions neutralize the difference between $\forall$-readings and $\exists$-readings by ruling out any scenarios in which this difference could be observed.

Although $\mathrm{HH}$ is formulated so as to apply only to adverbial quantifiers, Barker 
tentatively assumes that it governs nominal quantifiers as well. If so, the subjectasymmetric reading of example (16) presupposes that every man who owns several donkey beats all or none of them.

Most men who own a donkey beat it.

$\mathrm{HH}$ differs from the present account in that it predicts a presupposition failure for all those cases in which I assume a donkey sentence that is not literally true can be "true enough". An obvious challenge for $\mathrm{HH}$ arises from heterogeneous readings. Take sentence $(5 b)$, repeated here:

(17) Usually, if a man has a quarter in his pocket, he will put it in the meter.

We can predict its asymmetric $\exists$-reading based on these literal truth conditions:

a. true iff most quarter-owning men put all their quarters into the meter;

b. false iff at least one quarter-owning man puts no quarter into the meter;

c. neither in all other cases, for example, if every quarter-owning man puts exactly one quarter into the meter, and some of these men have additional quarters that they hold on to.

Let $w_{\text {true }}, w_{\text {false }}$, and $w_{\text {mixed }}$ be worlds described by (18a), (18b), and (18c) respectively. Suppose that the current issue is whether most men who have a quarter follow the law by putting at least one quarter into the meter. This is the case both at $w_{\text {true }}$ and at $w_{\text {mixed }}$. Hence (17) is true enough at $w_{\text {mixed }}$, and the present account will correctly predict that (17) on its asymmetric reading is interpreted heterogeneously as $\left\{w_{\text {true }}, w_{\text {mixed }}\right\}$, an $\exists$-reading.

By contrast, $\mathrm{HH}$ as presented so far wrongly rules out the asymmetric $\exists$-reading due to presupposition failure at $w_{\text {mixed }}$. Barker is aware of this and assumes that contextual domain narrowing prevents this presupposition failure by removing those quarters from consideration that remain in a man's pocket at $w_{\text {mixed }}$ after the parking laws have been satisfied. While Barker proposes no formal theory of domain narrowing, the general idea is that that any entities that do not settle the current issue can be removed from the domain. In the restricted domain, the homogeneity presupposition is satisfied, and (17) is predicted true.

In the absence of an explicit theory of domain narrowing, it is difficult to find examples for which Barker 1996 and the present account differ clearly in their predictions. One conceptual difference is that Barker assumes that homogeneity is a presupposition and that domain narrowing is always available to step in and rescue sentences from presupposition failure, while the present account does not treat donkey sentences as presuppositional and makes no assumptions about how domain narrowing works and under which conditions it is available. 
Homogeneity in donkey sentences

\section{A trivalent dynamic compositional semantics}

With a pragmatic theory in place that combines trivalent meanings with current issues to deliver disambiguated readings, the next step consists in delivering these trivalent meanings compositionally. As mentioned earlier, many early theories assumed that donkey pronouns can pick up both atoms and sums as discourse referents, so that the donkey pronoun in (4) could be paraphrased as the donkey or donkeys he owns (Lappin \& Francez 1994; Yoon 1994, 1996; Krifka 1996). But given the evidence in Kanazawa 2001 that singular donkey pronouns can only have atomic discourse referents, this is no longer an option.

Among those frameworks that do not interpret singular donkey pronouns as sum individuals, perhaps the most prominent and well-developed one is plural compositional discourse representation theory (PCDRT, Brasoveanu 2008, 2010). Accordingly, I will rely on PCDRT to generate and manage discourse referents.

PCDRT is an extension of CDRT (Muskens 1996), itself a reconstruction of Discourse Representation Theory (Kamp 1981). Various updates to the definition of PCDRT as given in Brasoveanu 2008 are described in Brasoveanu 2010 and other work in the same line of research. There are slight differences between the formulations of PCDRT in various papers and sometimes within the same paper. One difference concerns whether variable assignments are total, as in Brasoveanu 2008, or partial, as in Brasoveanu 2010. I will adopt partial assignments in order to be able to use the treatment of generalized quantifiers in Brasoveanu 2010, which is easier to extend to trivalent cases than the one in Brasoveanu 2008. This is not essential, though.

CDRT and PCDRT belong to the family of dynamic semantic systems. At the core of these systems is the notion of assignment. Assignments keep track of anaphora by relating discourse referents (drefs) $d, e, f$ etc. to entities $x, y, z$ etc. I let $v$ and $v^{\prime}$ range over drefs, and I write $d i$ for the value that the assignment $i$ assigns to the dref denoted by $d .{ }^{7}$ Partial assignments map drefs to the blank value $\star$.

In dynamic systems, constituents relate input states $I, I^{\prime}$ etc. to output states $O, O^{\prime}$ etc. While ordinary dynamic semantics and CDRT identify states with assignments, a PCDRT state is a set of assignments. This allows PCDRT to keep track of multiple assignments at once throughout the discourse. These assignments might, for example, correspond to multiple donkeys owned by the same man. The set of drefs is assumed to be fixed. Within a state, different assignments may agree on the values of certain drefs and disagree on the values of others. For example, a given state $S$ might consist of two assignments, which might map the dref $f$ to the same

7 Brasoveanu $(2008,2010)$ uses $u, u^{\prime}$ instead of $d, e, f$. Following Brasoveanu, I write $d i$ rather than the more intuitive $i(d)$ to reflect the fact that in PCDRT, assignments are taken as primitives rather than as functions, and discourse referents are taken to be functions from assignments to individuals. 
value - say, to the farmer Stevenson — and which might map the dref $d$ to different values - say, to the donkeys Modestine and Maxwelton, respectively.

Like many other dynamic theories, PCDRT assumes that anaphoric links are encoded in LFs through coindexation. Determiners are superscripted with the drefs they introduce, and anaphoric elements such as pronouns are subscripted with the drefs they pick up. For example, here is sentence (4) with annotations:

$$
\text { every }{ }^{f} \text { farmer who owns a }{ }^{d} \text { donkey beats } \text { it }_{d}
$$

Here, the distributive determiner every introduces the dref $f$, and the indefinite $a$ introduces the dref $d$ which is picked up by the donkey pronoun. The restrictor phrase of every relates every donkey-owning farmer $x$ to a state in which every assignment maps $f$ to $x$ and $d$ to a different donkey that $x$ owns. Suppose for example that we are in a model in which Stevenson is a farmer, and Modestine and Maxwelton are the donkeys he owns; and suppose that he beats Modestine but not Maxwelton. Then among the output states of (19), there will be the state $S$, whose assignments all map $f$ to Stevenson and $d$ either to Modestine or to Maxwelton.

In PCDRT, the notion of random assignment $i[d] o$ familiar from dynamic semantics, which expresses that $i$ and $o$ disagree at most in terms of the value they assign to $d$, is generalized as in (20).

$$
[d] \stackrel{\text { def }}{=} \lambda I \lambda O . \forall i \in I \exists o \in O . i[d] o \wedge \forall o \in O \exists i \in I . i[d] o
$$

In addition, I will make use of the notion of targeted assignment, which takes an input state $I$, a dref $d$, and an entity $x$ of arbitrary type, and modifies $I$ minimally so as to make sure that all of its assignments map $d$ to $x$ :

$$
[d:=x] \stackrel{\text { def }}{=} \lambda I \lambda O \cdot[d] I O \wedge \forall o \in O . d o=x
$$

As a shorthand, I will write $I[d] O$ for $[d] I O$ and $I[d:=x] O$ for $[d:=x] I O$.

We can restrict a state to assignments that map a given dref to nonblank values:

$$
I_{d \neq \star} \stackrel{\text { def }}{=}\{i \in I: d i \neq \star\}
$$

DRT and in its descendants distinguish between dynamic discourse representation structures (DRS) and static conditions. A DRS is a relation between states; a condition is a property of states. In PCDRT, lexical predicates are built around conditions that apply pointwise to each assignment. Nouns and verbs denote such predicates. In Brasoveanu 2010, only those assignments are considered that instantiate the relevant drefs with nonblank values. For example, the noun farmer denotes the predicate in (23a), and the transitive verb beats denotes the predicate in (23b).

$$
\text { a. } \quad \text { farmer } \rightsquigarrow \lambda v \lambda I \lambda O . I=O \wedge I_{d \neq \star} \neq \emptyset \wedge \forall i \in I_{d \neq \star} \text {. farmer }(v i)
$$


Homogeneity in donkey sentences

$$
\text { b. } \quad \text { beats } \rightsquigarrow \lambda v^{\prime} \lambda v \lambda I \lambda O . I=O \wedge I_{d \neq \star} \neq \emptyset \wedge \forall i \in I_{d \neq \star} \text {. beats }\left(v i, v^{\prime} i\right)
$$

As an example, suppose that $v=f$. Then the state $S$ from our earlier example will be an acceptable input state for farmer if every $i$ in $S$ that maps $f$ to a nonblank value maps it to a farmer. This is the case because Stevenson is a farmer.

Suppose now that $v^{\prime}=d$ in the same example. Then $S$ will be an acceptable input state for beats if every $i \in S$ that maps $f$ and $d$ to nonblank values maps them to two individuals such that the first beats the second. Following the conventions in (24), we abbreviate the predicates in (23) as in (25). These lexical entries do not produce any trivalent truth values yet; that part will be added later. Suppose (23b) is given an input state $I$ that represents a mixed scenario in that its assignments all map the dref $v$ to Stevenson and the dref $v^{\prime}$ to different donkeys of which Stevenson beats some but not others. Then (23b) will return false on this scenario. It will also return false, hovewer, if Stevenson beats none of these donkeys.

$$
\begin{array}{ll}
\text { a. } & R\{d\} \stackrel{\text { def }}{=} \lambda I . I_{d \neq \star} \neq \emptyset \wedge \forall i \in I_{d \neq \star} \cdot R(d i) \\
\text { b. } & {[C] \stackrel{\text { def }}{=} \lambda I \lambda O \cdot C(I) \wedge I=O} \\
\text { a. } & \text { farmer } \rightsquigarrow \lambda v .[\text { farmer }\{v\}] \\
\text { b. } & \text { beats } \rightsquigarrow \lambda v^{\prime} \lambda v .\left[\text { beats }\left\{v, v^{\prime}\right\}\right]
\end{array}
$$

PCDRT as originally defined (Brasoveanu 2008) assumes that a pronoun like $i t_{d}$ tests whether all assignments in the input set agree on the referent of $d$. I replace this by a weaker requirement that only checks whether each assignment maps $d$ to some atom, but not necessarily to the same value. This step paves the ground for the introduction of trivalence: Only if the values are allowed to differ can we capture the essence of a mixed scenario.

Like CDRT and like many other systems, PCDRT is a bivalent theory. That is, given a sentence, it always delivers an ordinary proposition. As we have seen, our pragmatic component expects a trivalent proposition. For this reason, we need to enrich it with a component which gives us trivalence by generating truth value gaps in mixed scenarios. There are different ways one could go about this. One possibility would be to partialize PCDRT in the sense of Muskens (1995) and Krahmer (1998). This would amount to making every PCDRT predicate potentially trivalent, including those that have nothing to do with the task at hand. Quantification over predicates would then have to include a special guard against trivalent predicates whenever they are not explicitly needed. This is definitely more trivalence than we need.

I will instead adopt a more localized approach. In the spirit of error state semantics (van Eijck 1993), I will enrich the system with special objects that can be passed around to signal that a mixed state has been encountered. Following a suggestion by Robert Henderson (p.c.), I will encode trivalence through a special 
discourse referent $\varepsilon$, which I will refer to as the error dref. By using the error dref, we can label states as mixed. This deviates from the original conception of error states in van Eijck 1993, where the error state contains no information besides the fact that an error has occurred. I assume that the error dref is always either mapped to $\star$ or to a designated error individual err. A value of $\star$ signals the absence of errors, while a value of err signals that an error has been encountered. I assume that all assignments map the error dref to $\star$ unless otherwise specified. I will call a state I errorful iff some $i \in I$ maps the error dref to err, and errorless otherwise. This can be defined in the object language as follows:

$$
\begin{array}{ll}
\text { a. } & \text { errorful } \stackrel{\text { def }}{=} \lambda I . \exists i \in I . \varepsilon i=e r r \\
\text { b. } & \text { errorless } \stackrel{\text { def }}{=} \lambda I . \neg \operatorname{errorful}(I)
\end{array}
$$

This setup allows us to mark individual assignments within a state by letting them map the error dref to err while letting others map it to $\star$. This allows us to distinguish farmers that beat all or none of their donkeys from other farmers within the same state that beat some but not all of their donkeys.

Given a DRS $D$ and a state $I$, let us say that $D$ succeeds on $I$ just in case $D$ relates $I$ to some non-errorful output state; $D$ fails on $I$ just in case $D$ does not relate $I$ to any output state at all; and $D$ returns an error on $I$ just in case $D$ relates $I$ to some output states but they are all errorful:

$$
\begin{array}{ll}
\text { a. } & \text { succeeds } \stackrel{\text { def }}{=} \lambda D \lambda I . \exists O . \text { errorless }(O) \wedge D I O \\
\text { b. } & \text { fails } \stackrel{\text { def }}{=} \lambda D \lambda I . \neg \exists O . D I O \\
\text { c. } & \text { error } \stackrel{\text { def }}{=} \lambda D \lambda I . \exists O . D I O \wedge \forall O . D I O \rightarrow \operatorname{errorful}(O)
\end{array}
$$

With these notions in place, it is easy to define negation, disjunction and conjunction of DRSs. Negation checks that every substate of a nonempty input state is errorless and leads to failure; disjunction offers a choice between running either disjunct; and conjunction threads the two conjuncts together sequentially:

$$
\begin{array}{ll}
\text { a. } & \sim D \stackrel{\text { def }}{=} \lambda I . I \neq \emptyset \wedge \text { errorless }(I) \wedge \forall H \subseteq I . \text { fails }(D)(H) \\
\text { b. } & D \mid D \stackrel{\text { def }}{=} \lambda I \lambda O . D I O \vee D^{\prime} I O \\
\text { c. } & D ; D \stackrel{\text { def }}{=} \lambda I \lambda O . \exists H . D I H \wedge D H O
\end{array}
$$

Since DRS negation is static in PCDRT, it is implemented as a condition. Conjunction and disjunction, however, have output states and are therefore themselves DRSs. While the definition of disjunction in Brasoveanu 2008 is static, my definition is dynamic. This will be useful in what follows.

The operation of maximalization is central to PCDRT. It takes a DRS $D$ and dref $d$ and modifies any input state so that its assignments store as many possible 
different entities under $d$ between them as possible as long as $D$ returns an output, errorless or not. The following definition is modified from Brasoveanu 2010 so as to allow for the possibility of errorful inputs (which are just passed on unchanged).

$$
\begin{aligned}
& \max ^{d}(D) \stackrel{\text { def }}{=} \lambda I \lambda O .(\operatorname{errorful}(I) \wedge I=O) \vee \\
& ([d] ; D) I O \wedge \neg \exists K .([d] ; D) I K \wedge O_{d \neq \star} \subset K_{d \neq \star}
\end{aligned}
$$

To create trivalent truth values, I assume that the pronoun $i t_{d}$ produces an error (by setting the error dref to err across all assignments) whenever its environment does not yield the same result for each value of $d$. For example, beats $i_{d}$ will take a subject argument $x$ and return an errorful state whenever the assignments in its input set map $u$ to different values and when $x$ beats some but not all of these values. The mixed state in our previous example will lead to such an errorful output state.

This is implemented by the following definitions. The test in (30a) takes a DRS $D$ (such as [beats $\{f, d\}]$ ) and a state, and checks whether either $D$ or its negation succeeds on this state. Since DRS negation is downward-entailing, this will fail on mixed states. The DRS in (30b) tags failed occurrences of (30a) as errorful. The term $I[\varepsilon:=e r r] O$ in (30b) uses targeted assignment to change $I$ into an errorful state.

$$
\begin{array}{ll}
\text { a. } & \text { uniformTest } \stackrel{\text { def }}{=} \lambda D \lambda I . \text { succeeds }(D)(I) \vee \operatorname{succeeds}([\sim D])(I) \\
\text { b. } \quad \text { uniform } \stackrel{\text { def }}{=} \lambda D \lambda I \lambda O .(\text { uniformTest }(D)(I) \wedge D I O) \vee \\
& (\neg \text { uniformTest }(D)(I) \wedge I[\varepsilon:=\text { err }] O)
\end{array}
$$

As for the pronoun $i t_{d}$, the basic lexical entry in (31) assumes that it occurs

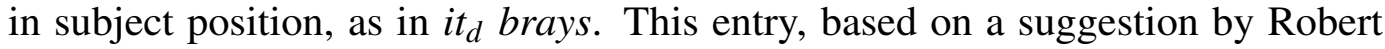
Henderson (p.c.), will succeed on any state $I$ if each assignment in $I$ maps $d$ to an entity that brays, and pass it on unchanged; if none of them do, it will fail; and if some but not all of them do, it will change $I$ to an errorful state. To understand the logic of (31), observe that its first component passes on a mixed state but marks it with an error, so that its second component passes it on without requiring that $P(d)$ holds in that state, as it would otherwise do.

$$
\text { it } \left.\left._{d} \rightsquigarrow \lambda P \text {. uniform }(P(d)) \text {; ([errorful }\right] \mid P(d)\right)
$$

The type shifter in (32a) is adapted from Heim \& Kratzer 1998. With its help, (31) combines with (25b) to yield the translation of beats $i_{d}$ in (32b). Given a dref $v$ (provided by the subject), this verb phrase will succeed on any input state $I$ if each assignment $i$ in $I$ maps $v$ and $d$ to entities $v i$ and $d i$ such that $v i$ beats $d i$; it will fail if none of them does; otherwise, it will flag each assignment of I with the error dref.

$$
\begin{aligned}
& \text { a. } \quad \text { Lift } \stackrel{\text { def }}{=} \lambda Q \lambda R \lambda v \cdot Q\left(\lambda v^{\prime} \cdot R\left(v^{\prime}\right)(v)\right) \\
& \text { b. } \quad \text { beats it } d \rightsquigarrow \lambda v . \text { uniform }([\text { beats }\{v, d\}]) ;([\text { errorful }] \mid[\text { beats }\{v, d\}])
\end{aligned}
$$


Let me now turn to the restrictor. Brasoveanu (2008) proposes that indefinites are ambiguous between a maximal or "strong" and a nonmaximal or "weak" interpretation. Maximal indefinites simultaneously introduce as many values as possible, while nonmaximal indefinites are free to assign a smaller set. For example, the assignments in any output state of $a^{d}$ donkey in (19) map $d$ to farmer-owned donkeys. If $a^{d}$ is maximal, these assignments do this in such a way that no farmer-owned donkey is left out. If $a^{d}$ is nonmaximal, among the output states of the indefinite there will be some whose assignments leave out some donkeys. In Brasoveanu 2008, the main purpose of this ambiguity is to account for the $\exists / \forall$ ambiguity. But this is no longer needed on the present theory. For this reason, I assume that indefinites always receive a maximal interpretation. Brasoveanu (2010), unlike Brasoveanu (2008), treats strong indefinites as anaphoric on the embedding quantifier. Because this complicates the relevant definitions, I stay with the entry taken from Brasoveanu 2008, slightly simplified here and shown in (33a). Without going into the details of the compositional semantics of the relative clause (see Brasoveanu 2008), the restrictor is assigned the interpretation in (33b):

$$
\begin{array}{ll}
\text { a. } & \mathrm{a}^{d} \rightsquigarrow \lambda P \lambda P^{\prime} \cdot \max ^{d}\left(P(d) ; P^{\prime}(d)\right) \\
\text { b. } \quad \lambda v \cdot[\text { farmer }\{v\}] ; \max ^{d}([\text { donkey }\{d\} ; \text { owns }\{v, d\}])
\end{array}
$$

The restrictor asks for a dref $v$ and an input state. It checks that every assignment in the input state maps $v$ to a farmer. It then instantiates a new dref $d$ and stores under it all the donkeys that $v$ owns. The dref is supplied by the embedding determiner. For example, if that determiner is every, it will quantify over farmers by first instantiating a dref with as many of these farmers as possible and then supplying the that dref as the value of the dref variable $v$. The determiner is also responsible for creating a separate state for each farmer. This is discussed at length in Brasoveanu 2010. Because the embedding determiner needs to manage quantificational dependencies and states, the definition given for it in Brasoveanu 2010 is somewhat involved, as we will see below. At its heart, though, is a static determiner, and this is the only component we need to manipulate.

I make embedding determiners supervaluationist on their second argument in the sense of van Eijck (1996). A supervaluation quantifier when applied to an errorgenerating predicate (such as beats it) returns a classical truth value whenever the underlying bivalent quantifier returns the same truth value no matter how error states are resolved, and returns Neither (encoded as an error) otherwise. For example, Every farmer ${ }^{f}$ who owns $a^{d}$ donkey when applied to beats it ${ }_{d}$ yields the trivalent truth conditions in (8a), and similarly for the other sentences we have seen.

The main idea in the implementation to follow is that processing the restrictor farmer $^{f}$ who owns $a^{d}$ donkey and the nuclear scope beats it ${ }_{d}$ gives access to two 
sets. The "inner envelope" contains every donkey-owning farmer who beats all of his donkeys. The "outer envelope" contains every donkey-owning farmer who beats at least one of his donkeys. A supervaluation quantifier succeeds if the underlying ordinary quantifier is true of all sets that are supersets of the inner envelope and subsets of the outer envelope; it fails if the underlying ordinary quantifier is false of all these sets; otherwise, it produces an error. This is implemented in (34) and (35). Some auxiliary definitions follow. The definitions in (36) are motivated and explained in Brasoveanu 2010. Definition (37) specifies the sets that are sandwiched between the inner envelope $f I_{\varepsilon=\star}$ and the outer envelope $f I$ associated with $f$.

The entry (34), slightly modified from Brasoveanu 2010, first stores under $f$ all those individuals that satisfy the restrictor and then copies from $f$ into $f^{\prime}$ all those individuals that also satisfy the nuclear scope. Finally, $f$ and $f^{\prime}$ are passed to the supervaluationist determiner, defined as in in (35). Here, the input state is passed on unchanged if the underlying determiner accepts all precisifications of the nuclear scope; if the determiner accepts some but not all of these precisifications, an error is generated. This makes the determiner behave supervaluationistically.

$$
\operatorname{det}^{f} \rightsquigarrow \lambda P \lambda P^{\prime} \cdot \max ^{f}\left({ }_{\langle f\rangle} P(f)\right) ; \max ^{f^{\prime}}\left(\left[f^{\prime} \sqsubseteq f\right] ;\left\langle f^{\prime}\right\rangle P^{\prime}\left(f^{\prime}\right)\right) ; \operatorname{DET}_{s v}\left\{f, f^{\prime}\right\}
$$

Let DET be a static determiner. Then $\operatorname{DET}_{s v}\left\{f, f^{\prime}\right\} \stackrel{\text { def }}{=} \lambda I \lambda O$.

$\left(\left(\forall S \in \operatorname{sv}\left(f^{\prime}, I\right)\right.\right.$. DET $\left.\left.(f I, S)\right) \wedge I=O\right) \vee$

$\left(\left(\exists S \in \operatorname{sv}\left(f^{\prime}, I\right) . \operatorname{DET}(f I, S) \wedge \neg \forall S \in \operatorname{sv}\left(f^{\prime}, I\right) . \operatorname{DET}(f I, S)\right) \wedge I[\varepsilon:=e r r] O\right)$

$$
\begin{array}{ll}
\text { a. } & \text { Image of } I \text { under } f: \\
& f I \stackrel{\text { def }}{=}\{f i: i \in I\} \backslash\{\star\} \\
\text { b. } \quad \text { Structural inclusion: } & f^{\prime} \sqsubseteq f \stackrel{\text { def }}{=} \lambda I . \forall i \in I . f^{\prime} i=f i \vee\left(f^{\prime} i=\star \wedge \forall i^{\prime} \in I . f^{\prime} i^{\prime} \neq f i\right) \\
\text { c. } \quad \text { Distributivity operator: } & \langle f\rangle \stackrel{\text { def }}{=} \lambda I \lambda O . I_{f=\star}=O_{f=\star} \wedge(f I=\emptyset \rightarrow I=O) \wedge \\
& \left(f I \neq \emptyset \rightarrow\left(f I=f O \wedge \forall x . x \in f I \rightarrow D I_{f=x} O_{f=x}\right)\right) \\
\operatorname{sv}(f, I) \stackrel{\text { def }}{=}\left\{S \mid f I_{\varepsilon=\star} \subseteq S \subseteq f I\right\}
\end{array}
$$

\section{Conclusion}

Definite plurals and donkey sentences can be given a uniform pragmatic treatment. Previous accounts relied on the problematic assumption that it and the donkey $(s)$ he owns can be given a parallel analysis in terms of plural individuals. However, Kanazawa (2001) showed that plural individuals cannot be involved in the semantics of $i$. This paper avoids the need for plural individuals by leveraging PCDRT's evaluation-level pluralities. Aided by error-state and supervaluation semantics, PCDRT delivers trivalent semantics to the pragmatics in a fully compositional way. 
Champollion

\section{References}

Barker, Chris. 1996. Presuppositions for proportional quantifiers. Natural Language Semantics 4(3). 237-259. doi:10.1007/bf00372821.

Brasoveanu, Adrian. 2008. Donkey pluralities: Plural information states versus non-atomic individuals. Linguistics and Philosophy 31(2). 129-209. doi:10.1007/s10988-008-9035-0.

Brasoveanu, Adrian. 2010. Decomposing modal quantification. Journal of Semantics 27(4). 437-527. doi:10.1093/jos/ffq008.

Chierchia, Gennaro. 1992. Anaphora and dynamic binding. Linguistics and Philosophy 15(2). 111-183. doi:10.1007/bf00635805.

Chierchia, Gennaro. 1995. Dynamics of Meaning: Anaphora, Presupposition, and the Theory of Grammar. Chicago, IL: University of Chicago Press. doi:10.7208/chicago/9780226104515.001.0001.

Dekker, Paul. 1993. Transsentential meditations: Ups and downs in dynamic semantics: University of Amsterdam PhD dissertation. http://hdl.handle.net/ 11245/1.392554.

van Eijck, Jan. 1993. The dynamics of description. Journal of Semantics 10(3). 239-267. doi:10.1093/jos/10.3.239.

van Eijck, Jan. 1996. Quantifiers and partiality. In Jaap van der Does \& Jan van Eijck (eds.), Quantifiers, Logic, and Language, 105-144. Stanford, CA: CSLI Publications.

van Fraassen, Bas C. 1969. Presuppositions, supervaluations and free logic. In Karel Lambert (ed.), The Logical Way of Doing Things, chap. 4, 67-91. New Haven, CT: Yale University Press.

Gawron, Jean Mark, John Nerbonne \& Stanley Peters. 1991. The absorption principle and E-type anaphora. In Jon Barwise, Jean Mark Gawron, Plotkin \& Syun Tutiya (eds.), Situation Theory and its Applications 2, 335-362. Saarbrücken, Germany: CSLI Publications. http://scidok.sulb.uni-saarland.de/volltexte/2011/3581.

Geurts, Bart. 2002. Donkey business. Linguistics and Philosophy 25(2). 129-156. doi:10.1023/a:1014624331450.

Groenendijk, Jeroen \& Martin Stokhof. 1984. Studies on the semantics of questions and the pragmatics of answers. Amsterdam, Netherlands: University of Amsterdam $\mathrm{PhD}$ dissertation.

Heim, Irene. 1982. The semantics of definite and indefinite noun phrases. Amherst, MA: University of Massachusetts PhD dissertation. http://semanticsarchive.net/ Archive/jA2YTJmN.

Heim, Irene. 1990. E-type pronouns and donkey anaphora. Linguistics and Philosophy 13(2). 137-178. doi:10.1007/bf00630732.

Heim, Irene \& Angelika Kratzer. 1998. Semantics in Generative Grammar. Oxford, 
Homogeneity in donkey sentences

UK: Blackwell Publishing.

Kamp, Hans. 1981. A theory of truth and semantic representation. In Jeroen Groenendijk, Theo Janssen \& Martin Stokhof (eds.), Formal Methods in the Study of Language 135 Mathematical Center Tracts, 277-322. Amsterdam, Netherlands: Mathematisch Centrum. doi:10.1002/9780470758335.ch8.

Kanazawa, Makoto. 1994. Weak vs. strong readings of donkey sentences and monotonicity inference in a dynamic setting. Linguistics and Philosophy 17(2). 109-158. doi:10.1007/bf00984775.

Kanazawa, Makoto. 2001. Singular donkey pronouns are semantically singular. Linguistics and Philosophy 24(3). 383-403. doi:10.1023/a:1010766724907.

Krahmer, Emiel J. 1998. Presupposition and anaphora. CSLI Lecture Notes 89. Stanford, CA: CSLI Publications. https://pure.uvt.nl/portal/files/759751/ presupposition-and-anaphora-book.pdf.

Krifka, Manfred. 1996. Pragmatic strengthening in plural predications and donkey sentences. In Teresa Galloway \& Justin Spence (eds.), Semantics and Linguistic Theory Conference (SALT) 6, 136-153. Ithaca, NY: Cornell University CLC Publications. doi:10.3765/salt.v6i0.2769.

Križ, Manuel. 2016. Homogeneity, non-maximality, and all. Journal of Semantics 33(3). 493-539. doi:10.1093/jos/ffv006.

Lappin, Shalom \& Nissim Francez. 1994. E-type pronouns, i-sums, and donkey anaphora. Linguistics and Philosophy 17(4). 391-428. doi:10.1007/bf00985574.

Lasersohn, Peter. 1999. Pragmatic halos. Language 75(3). 522-551. doi:10.2307/417059.

Lewis, David. 1979. Scorekeeping in a language game. Journal of Philosophical Logic 8(1). 339-359. doi:10.1007/bf00258436.

Löbner, Sebastian. 2000. Polarity in natural language: Predication, quantification and negation in particular and characterizing sentences. Linguistics and Philosophy 23(3). 213-308. doi:10.1023/a:1005571202592.

Malamud, Sophia A. 2012. The meaning of plural definites: A decision-theoretic approach. Semantics and Pragmatics 5(3). 1-58. doi:10.3765/sp.5.3.

Muskens, Reinhard. 1995. Meaning and partiality. Stanford, CA: CSLI Publications.

Muskens, Reinhard. 1996. Combining Montague semantics and discourse representation. Linguistics and Philosophy 19(2). 143-186. doi:10.1007/bf00635836.

Roberts, Craige. 2012. Information structure in discourse: Towards an integrated formal theory of pragmatics. Semantics and Pragmatics 5(6). 1-69. doi:10.3765/sp.5.6.

van Rooij, Robert. 2003. Questioning to resolve decision problems. Linguistics and Philosophy 26(6). 727-763. doi:10.1023/b:ling.0000004548.98658.8f.

Rooth, Mats. 1987. Noun phrase interpretation in Montague Grammar, File Change Semantics, and Situation Semantics. In Peter Gärdenfors (ed.), Generalized 
quantifiers: Linguistic and Logical Approaches, 237-268. Dordrecht, Netherlands: Reidel.

Schubert, Lenhart K. \& Francis Jeffry Pelletier. 1989. Generically speaking, or, using discourse representation theory to interpret generics. In Gennaro Chierchia, Barbara H. Partee \& Raymond Turner (eds.), Properties, Types, and Meaning, Volume II: Semantic Issues 39 Studies in Linguistics and Philosophy, 193-268. Dordrecht, Netherlands: Kluwer. doi:10.1007/978-94-009-2723-0_6.

Schwarzschild, Roger. 1993. Plurals, presuppositions and the sources of distributivity. Natural Language Semantics 2(3). 201-248. doi:10.1007/bf01256743.

Yoon, Youngeun. 1994. Weak and strong interpretations of quantifiers and definite NPs in English and Korean: University of Texas at Austin PhD dissertation.

Yoon, Youngeun. 1996. Total and partial predicates and the weak and strong interpretations. Natural Language Semantics 4(3). 217-236. doi:10.1007/bf00372820.

Lucas Champollion

Department of Linguistics

New York University

10 Washington Place

New York, NY 10003

champollion@nyu.edu 